\title{
Virtual holographic laboratory
}

\author{
M. L. Calvo, T. Alieva, J. Rodrigo, O. Martínez-Matos, A. \\ Moreno, et al.
}

M. L. Calvo, T. Alieva, J. A. Rodrigo, O. Martínez-Matos, A. Moreno, T. Aliev, "Virtual holographic laboratory," Proc. SPIE 9665, Tenth International Topical Meeting on Education and Training in Optics and Photonics, 96651R (3 June 2007); doi: 10.1117/12.2207686

SDIE Event: Tenth International Topical Meeting on Education and Training in Optics and Photonics, 2007, Ottawa, Ontario, Canada 


\title{
Virtual Holographic Laboratory
}

\author{
M. L. Calvo, T. Alieva, J. A. Rodrigo, O. Martínez-Matos, A. Moreno, T. Aliev² \\ Departamento de Óptica, Facultad de Ciencias Físicas, Universidad Complutense de Madrid, 28040 Spain, \\ 2.- Polytechnic School, Universidad Autónoma de Madrid, Canto Blano, Madrid, Spain \\ mlcalvo@fis.ucm.es
}

\begin{abstract}
In this work we present a Virtual Holographic Laboratory for educational purposes. This project is edited on DVD support and it has been designed to be interactive: schemes, pictures, videos in order to clarify the theoretical description of the phenomena improving the understanding of its fundamental concepts. We believe that this project is helpful for undergraduate and graduate students in physics and engineering to obtain the solid knowledge about holography and to prepare for practical lessons on holography or partially substitute the lasts in the case of absence of appropriated technical base at a specific university level.
\end{abstract}

\section{Introduction}

Nowadays holography is playing an important role in science and technology. Holographic recording and reconstruction of an object is probably the most illustrative example of various optical phenomena and concepts such as interference, diffraction, coherence, etc. Moreover holography is very attractive to the students and therefore to motivate them toward to the entire area of optics and photonics. These are the main reasons which yield to including the holography in the curriculum of many universities over the world. Certainly the practical lessons on holography are needed for deeper understanding. Nevertheless some universities in the developing countries cannot afford them. We have recently created a computer assisted course ${ }^{1}$ dedicated to analogue and digital holography which can partially substitute the practical lessons. The course edited on DVD (HTMLbased) is written in Spanish and its contents correlate with the Master courses on Statistical Optics and Holography given at the Faculty of Physics of Complutense University of Madrid (UCM).

The DVD contains:

- Historical introduction to holography, including links to the Nobel Prize lecture of its founder D. Gabor.

- Fundamentals of holography where the phenomena of interference and diffraction as well as concepts of coherence are discussed.

- Description of various schemes and methods for holographic recording and reconstruction.

- Special section dedicated to digital holography where digital writing and reading processes are discussed.

- Description and classification of materials and devices for holographic recording.

- Discussion of application of holography in science, technology and every day life.

- Three designed practical lessons, corresponding to the course of Statistical Optics at UCM.

- References and links.

In the following section we present a brief resume of the contents of the Project by enhancing the principal objectives.

Tenth International Topical Meeting on Education and Training in Optics and Photonics, edited by Marc Nantel, Proc. of SPIE Vol. 9665, 96651R - (c) 2007 SPIE, OSA, IEEE, ICO doi: $10.1117 / 12.2207686$ 


\section{Overview of the project}

The edition of this Project is under HTML format and multimedia contents as Flash-Video, then allowing its use in the most popular operative systems: MS Windows, Mac OSX and Linux. This design provides a complete integration with the common web navigators (Web Browser) and at the same time it turns out to be quite familiar to the user. This allows also to incorporating various links to scientific papers via Internet. The various menus have been designed to perform a quick and easy access to the various DVD's sections and contents.

The previous use of this material in practical courses has demonstrated to be a tool of great applicability at the time to afford the preparation of the undergraduate students for laboratory interaction as well as being quite useful for concepts assimilation.

\subsection{Historical introduction to holography.}

The reader has access to each one of the sections in an independent manner and without the requirement to accomplish a predetermined order. However, it is advisable, and in particular in the case of fresh students, to follow the proposed sequence of sections, as indicated in the menu in order to assure a sequential logical and gradual learning.

The first section is dedicated to a historical introduction of the holography as well as a presentation of the importance and technological impact of this science. As well as for the rest of the sections and at the end, it is facilitated a number of bibliographic references to orient those students interested in a deeper approach of the subject. This section then is also an introductory basis to the most characteristic aspects of the holography.

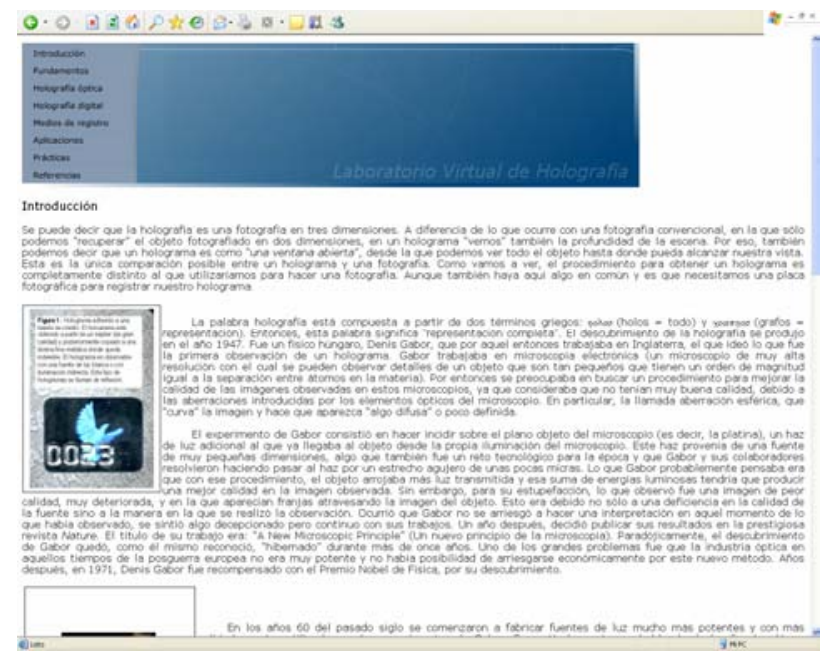

Figure 1 (color on line).- Screenshot corresponding to the historical introduction to holography. 


\subsection{Fundamentals of holography}

The objective of this part of the project is to facilitate to the student the assimilation on the main processes characterizing the recording and reading of a hologram. Concepts such as real and virtual images of the holographically recorded object are usually not very well understood and introduce some confusion in the interpretation by fresh students. Therefore, these concepts are specially treated and illustrated with profusion of pertinent graphics and schemes (as it is done for the rest of the sections).

This section is divided into three parts clearly differentiated: an introduction to the principles of interference and diffraction of light, definition and types of holograms and finally the requirements of coherence of light for the recording and reading of holograms.

In each one of these parts it is presented a well detailed and concise explanation of the key aspects involved in the recording and reading of a hologram. Interactive videos demonstrating phenomena such as interference and diffraction of light by various objects are available and help to illustrate the basic concepts to the student. Usually, in a room lecture, students are affording the typical mathematical description, so that these multimedia materials are interesting complements to the teacher. Figure 2 shows some of these illustrations.

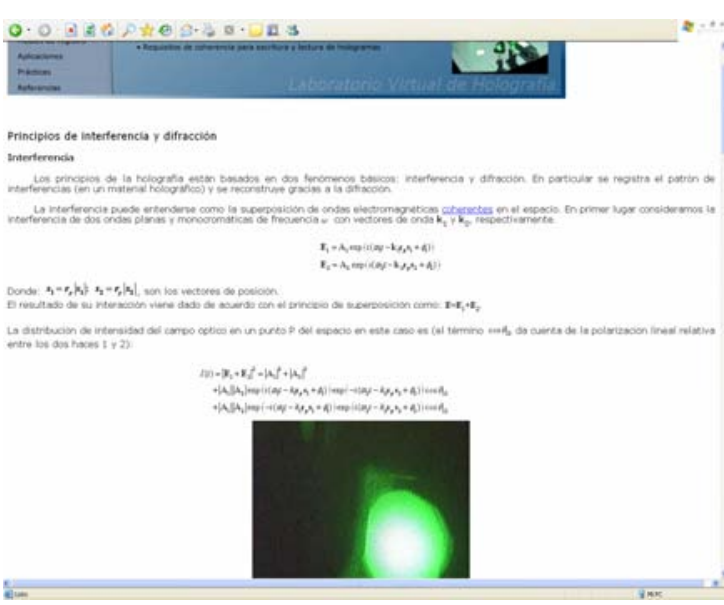

(a)

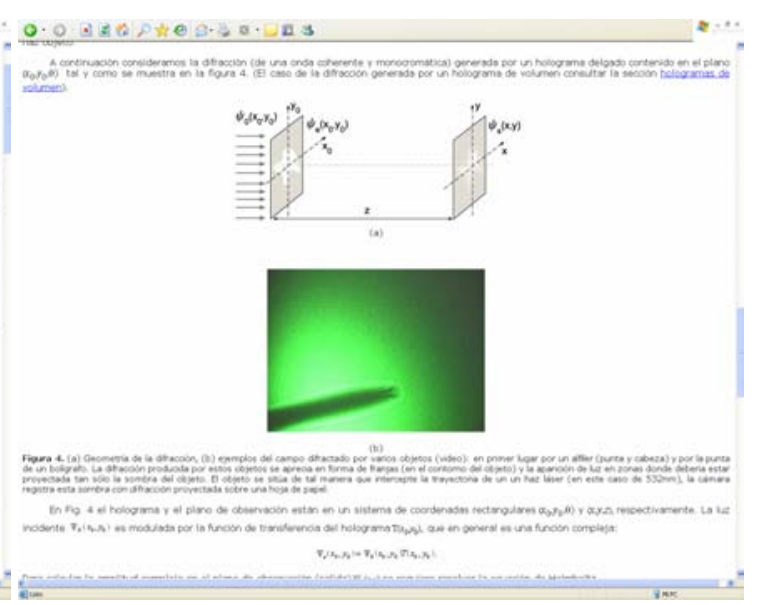

(b)

Figure 2 (color on line).- Interference (a) and diffraction (b) sections where different examples are introduced. Flash videos for these examples are also displayed.

Accordingly, the mathematical description describing the various processes for the recording and reading of the hologram is introduced as well. Various schemes for the study of the various types of holograms, say, for example, reflection and transmission ones are also displayed (see Figure 3). 


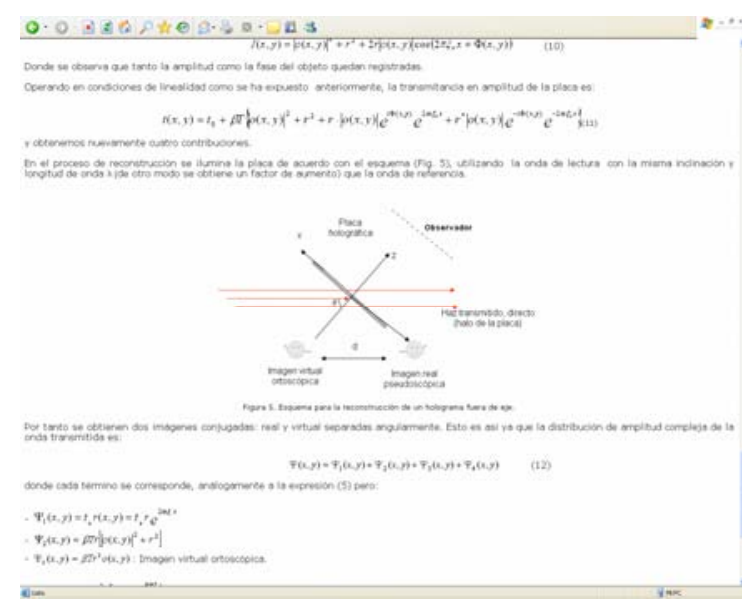

(a)

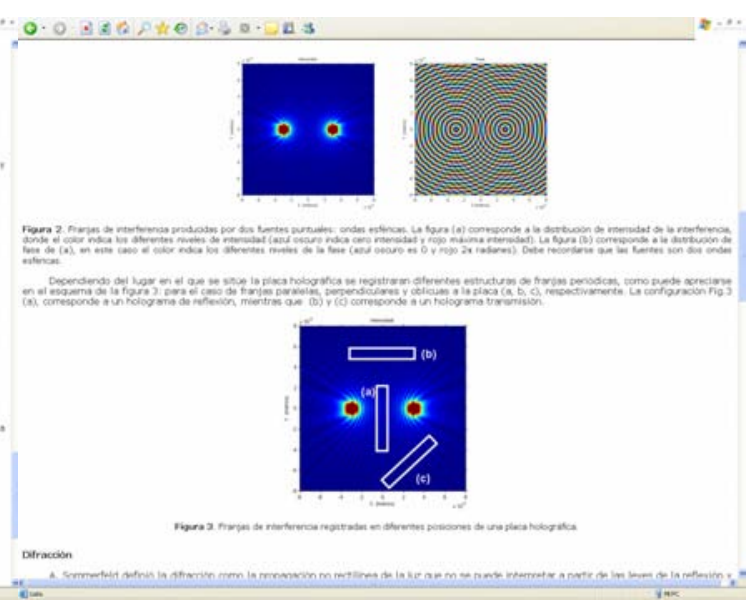

(b)

Figure 3 (color on line).- Transmission and reflection holograms illustrated by means of different figures, for instance (a) and (b).

Moreover, it is provided a mathematical description of the differences arising between real and virtual images at the time of reading the hologram. These concepts are treated as well in the following sections and providing interactive videos. Finally, it is discussed the requisites regarding the spatial and temporal coherence of the optical beam, as key factors for obtaining high quality holograms.

\subsection{Holography: recording and reading schemes, types of holograms, observation and hologram quality.}

As explained above, one of the main objectives is that the student be capable to distinguish the various types of holograms and the differences arising between real and virtual images regarding the kind of contained information. This section reinforce the knowledge previously introduced and by mean of practical examples.

The section presents various schemes for the recording and reading procedures (see Figure 4) as well as the definition of thick and thin holograms, respectively.

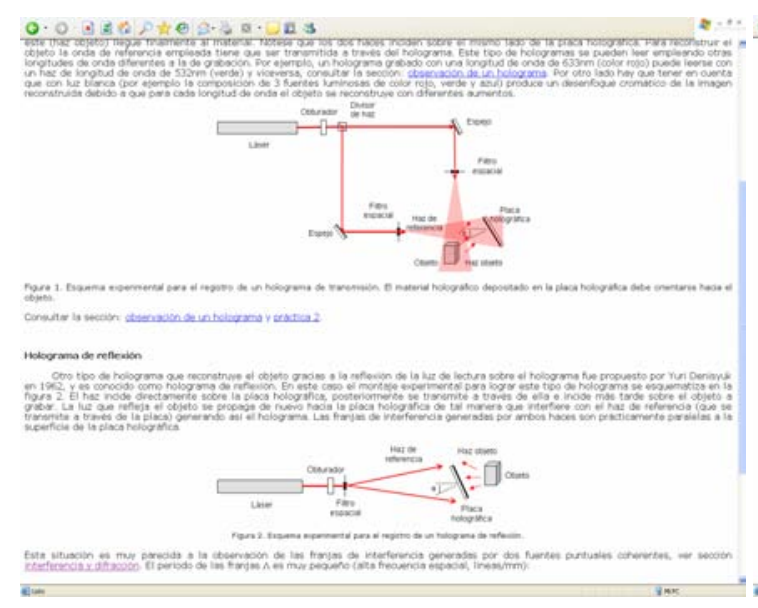

(a)

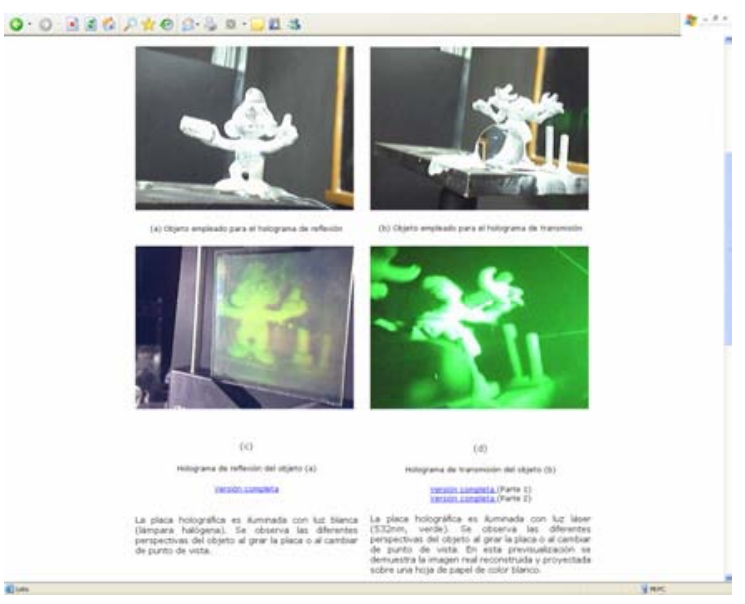

(b)

Figure 4 (color on line).- (a) Schemes and methods for holographic recording and their experimental implementation are discussed. The hologram reconstruction is shown as a Flash-Video for different points of view, (b). 
More insights are given in Figure 4(b) with the aim to illustrate the differences between real and virtual images. Here the interactive multimedia provides a direct observation of both images. This is indeed a very useful tool for the understanding of concepts then facilitating the student task.

\subsection{Digital holography: digital writing and reading processes.}

One of the main objectives of the present project is to afford the introduction of advanced concepts and techniques that are being currently applied in current research subjects. This is part of a new and attractive work planning to the student. In particular, in this section it is studied with detail the techniques for the recording and reading of 2-D digital holograms (see Figure 5). Moreover, the various experimental schemes to be used for this task are displayed with an emphasis on the characteristics of Spatial Light Modulators (SLM), CCD cameras and CMOS. The section is devoted as well to the study of the technical aspects that correspond to the specifications of these devices. The 3-D holographic technique is presented by including an up dated list of references.

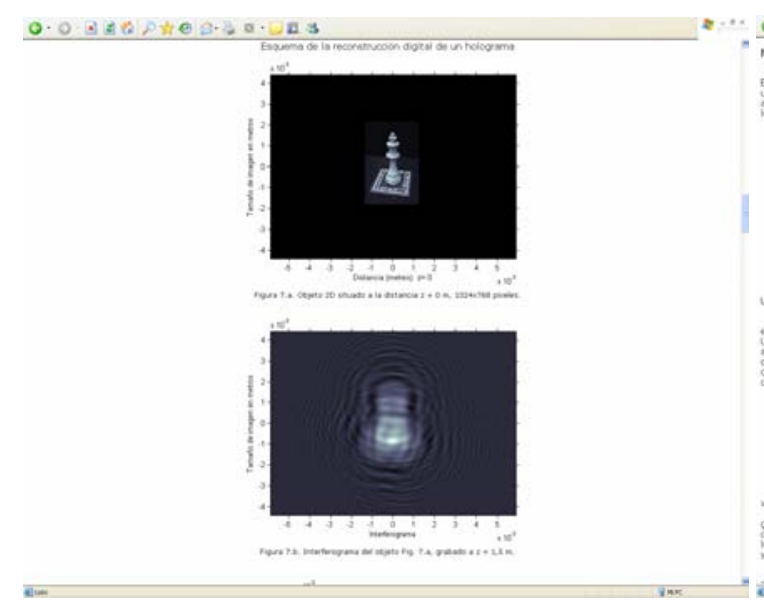

(a)

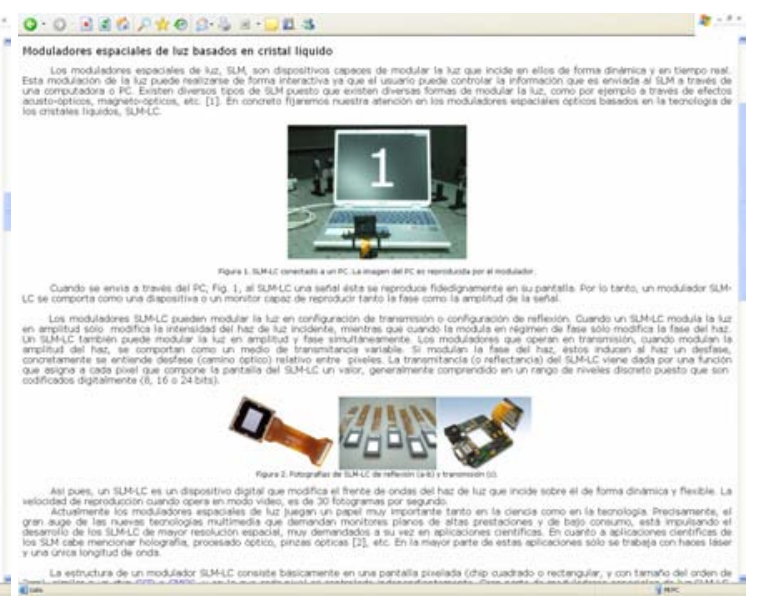

(b)

Figure 5 (color on line).- Digital hologram section, (a). Spatial Light Modulator and CCD devices are also introduced in this section, (b).

The section devoted to 2-D digital holography includes the concepts of recording and reading of Fresnel holograms: computer generated and optically reproduced via SLM. Also, the optically recording procedure is explaining with the use of the CCD and post computational reproduction. To complete with the study various schemes are displayed as well as numerical computation results. It is introduced practical work as for example the reconstruction of different parts of a digital interferogram with the aim to show the contained codified information. 


\subsection{Description and classification of materials and devices for holographic recording.}

An important aspect to introduce is the classification of the various types of holographic materials as well as its technological applications. In particular, it is presented a detailed discussion of the most relevant current holographic materials and the advantages presented in its used (see Figure 6). It is mentioned the organic and inorganic photorefractive materials as well as the photopolymerizables glasses, originally synthesized in our laboratory ${ }^{2}$.

Foundations of the techniques such as multiplexing and concepts such as scattering are studied as well. As a technological application we have included a detailed description of the holographic data storage (see Figure 6(b)).

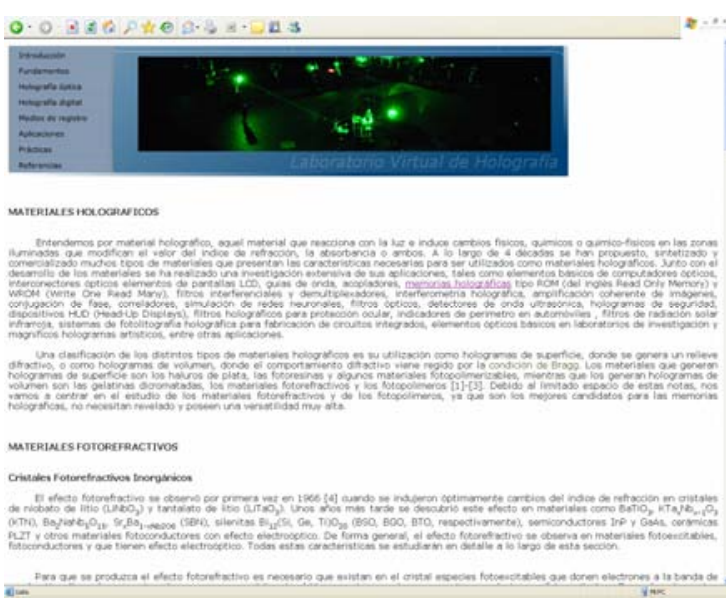

(a)

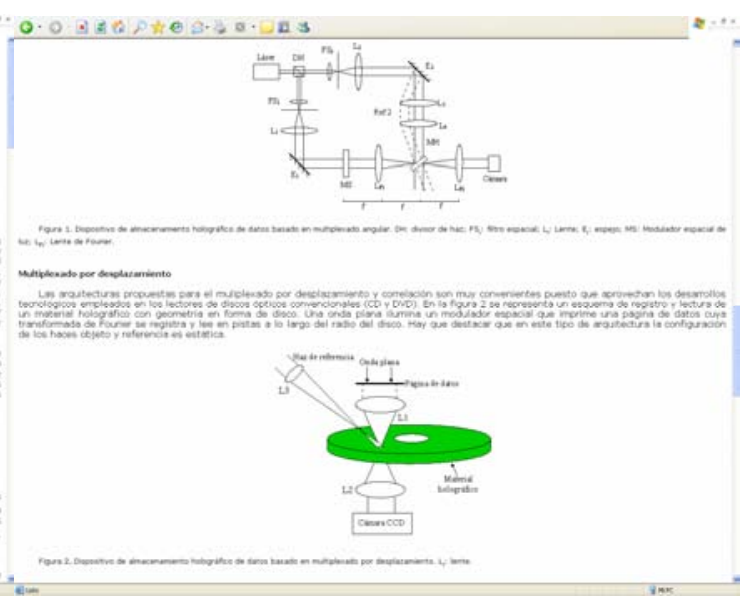

(b)

Figure 6 (color on line).- Description and classification of different holographic materials are presented, (a). Applications such as holographic data storage are also discussed, (b).

\subsection{Discussion of application of holography in science, technology and every day life.}

In this section it is described some of the most relevant and appealing technological applications of holography in science and daily life. It includes cases as encryption systems (see Figure 7 a-b) as well as applications in biomedicine. 


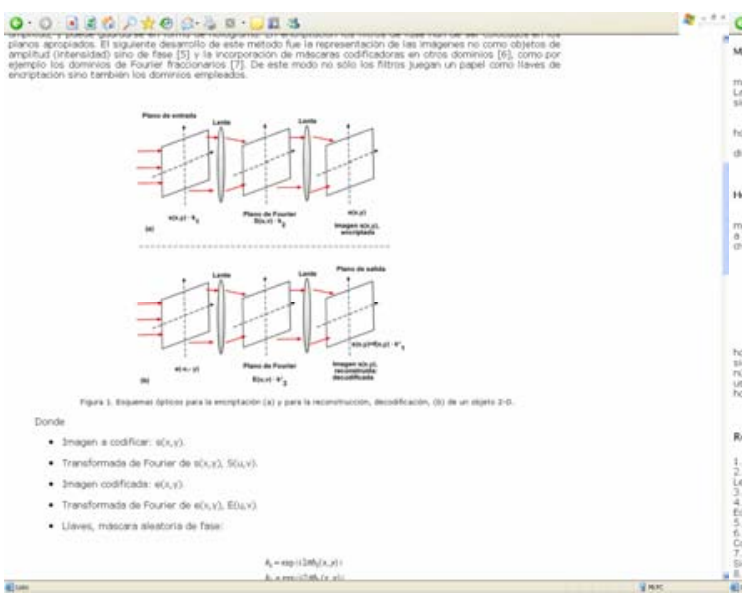

(a)

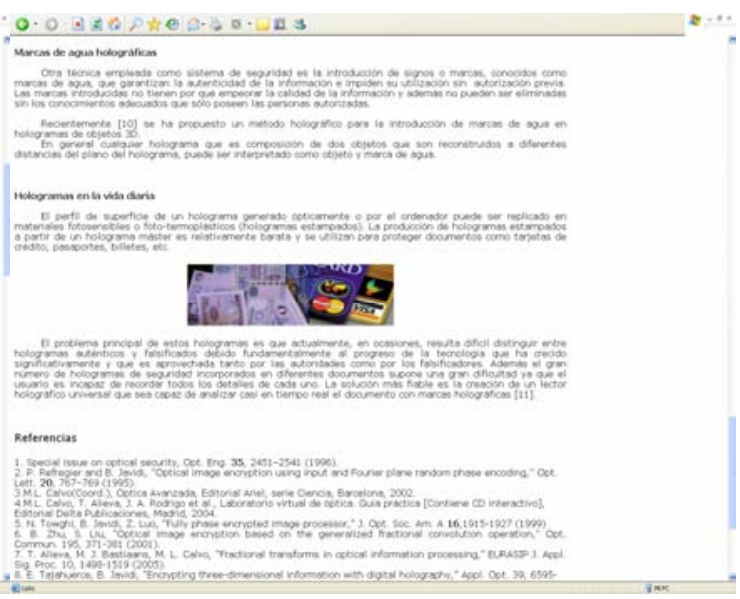

(b)

Figure 7 (color on line).- Conventional encryption techniques (a) as well as different holographic applications in biomedicine, technology and security systems are discussed (b).

We believe this constitutes a good example to the student on the various nowadays perspectives of holography development.

\subsection{Laboratory practical lessons: reflection and transmission holograms recording and numerical simulation of 2-D digital holograms.}

In the precedent sections we have presented a brief description of the contents of the project. The last section is dedicated to the proposal of three practical experiences in the laboratory with the aim to introduce to the student on the various technical procedures for recording and reading of holograms in both aspects analogical and digital, respectively.

The basic idea is to give to the students a series if instructions so that they can be ready to afford their personal work in the laboratory. Thus, the various experimental set-up are displayed, interferometers, spatial filtering and chemical procedure for developing the BB-640 holographic plates used for the practical work.

One important aspect included as well is the mentioning of all the security rules to be applied regarding the use of a laser beam and chemical products. The student has to be able to record reflection and transmission holograms and object reconstruction (see Figure 8.). 


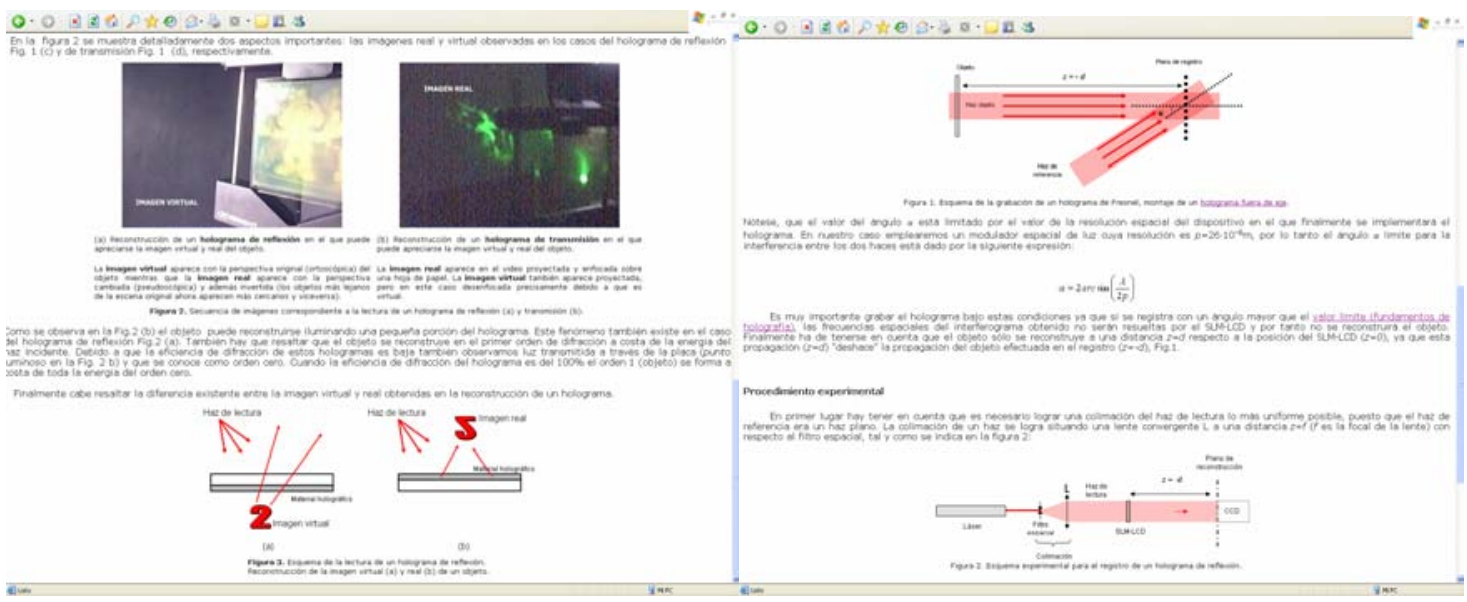

(a)

(b)

Figure 8 (color on line).- Practical lessons for analogue and digital holography, (a) and (b) respectively.

In the practical work for 2-D digital holography the student has to perform various Fresnel holograms with the use of a personal computer and reconstruct it optically via SLM. The multiplexing techniques in digital holography are proposed to the student as an advanced problem.

We stress that the presented guide is useful for both students and teachers since it contains general and detailed information on practical holography that can be afforded as well by amateurs.

\subsection{References and links.}

The guided ends with a list of references organized in categories as: text books, scientific articles and links to web sites dedicated to holography.

\section{Conclusion}

The structure of the project has been oriented to obtain a gradual assimilation of concepts by the student. Also, it has been introduced in a detailed way so that advanced readers can also benefit of the contents. The multimedia material included is an important tool for a dynamical learning. Special emphasis is dedicated to carefully designed schemes (recording and reading), types of analogical and digital holograms, described in a very concise fashion. The physical concepts as diffraction of light and interferences are studied in detail. The discussion on the various current technological applications, holographic materials as well as optoelectronic devices (SLM, CCD, etc.) provides to the student and completes knowledge and enhances the motivation.

The proposed guide has been used during the academic course 2006/2007 in the Holographic Laboratory associated to the Statistical Optics subject. This is part of subjects studied by undergraduate students in fundamental physics (fourth course). A total number of thirty six students divided into ten groups were working in practical holography. The results were indeed a great success showing the great acceptation of this kind of didactical method. Some of these students are now pursuing advanced subjects in holography under the guidance of the teachers of this project. 


\section{Acknowledgements}

This Project has been financed by the Vice-Rectorate of Innovation and European Space of High Education

Degrees, Office of European Convergence, Complutense University of Madrid (Project 303/2005).

\section{References}

[1]. M. L. Calvo, T. Alieva, J. A. Rodrigo, O. Martinez Matos, A. Moreno, T. Aliev, "Holografía. Laboratorio Virtual” (A virtual laboratory of holography.), Ed. UCM, Madrid (November 2007, CD in Spanish).

[2].- P. Cheben, "Materiales de registro para almacenamiento óptico de datos" (Recording materials for optical data storage), in: Óptica Avanzada (Advanced Optics), (M.L. Calvo, coord.), Ed. Ariel Ciencia, Barcelona, 2002, Chapter 10 